\title{
Phase transitions in the extended particle systems, Hagedorn temperature and fractal dimension of space, as a confinement phase transition order parameter
}

\author{
Nugzar Makhaldiani ${ }^{1, *}$ \\ ${ }^{1}$ Joint Institute for Nuclear Research, 141980 Dubna Moscow region, Russia
}

\begin{abstract}
We consider a formal definition of New physics, fundamental constants of physics, formulation of the quantum statistical theory including extra integrals, monopole mechanism for hadronization in $\mathrm{QCD}$, finite approximation of the zeta-function and fermion factorization of the bosonic statistical sum.
\end{abstract}

I always knew that sooner or later $\mathrm{p}$ adic numbers will appear in Physics.

André Weil.

We say that we find New Physics (NP) when either we find a phenomenon which is forbidden by Standard Model (of elementary particles) (SM) in principal - this is the qualitative level of NP - or we find a significant deviation between precision calculations in SM of an observable quantity and a corresponding experimental value.

In the Universe, matter has mainly two geometric structures, homogeneous, and hierarchical. The homogeneous structures are naturally described by real numbers with an infinite number of digits in the fractional part and usual archimedean metrics. The hierarchical structures are described with p-adic numbers with an infinite number of digits in the integer part and non-archimedean metrics [1]. A discrete, finite, regularized version of the homogeneous structures are homogeneous lattices with constant steps and distance rising as arithmetic progression. The discrete version of the a hierarchical structures is hierarchical lattice-tree with a scale rising in geometric progression.There is an opinion that the present day theoretical physics needs (almost) all mathematics, and the progress of modern mathematics is stimulated by fundamental problems of theoretical physics.

In the 1870's G.J. Stoney, [2] the physicist who coined the term "electron" and measured the value of elementary charge $e$, introduced as universal units of Nature for $L, T, M$ :

$$
l_{S}=\frac{e}{c^{2}} \sqrt{G}, t_{S}=\frac{e}{c^{3}} \sqrt{G}, m_{S}=\frac{e}{\sqrt{G}}
$$

M. Planck, [3] introduced as universal units of Nature

$$
m_{P}=\sqrt{\frac{h c}{G}}=\frac{m_{S}}{\sqrt{\alpha}}, l_{P}=\frac{h}{c m_{P}}=\frac{l_{S}}{\sqrt{\alpha}}=11.7 l_{S}, t_{P}=\frac{l_{P}}{c}=\frac{t_{S}}{\sqrt{\alpha}}
$$

\footnotetext{
*e-mail: mnv@jinr.ru
} 
Stoney's fundamental constants are more fundamental just because they are less than Planck's constants. Due to the value of $\alpha^{-1}=137$, we can consider relativity theory and quantum mechanics as deformations of the classical mechanics when deformation parameter $c=137$ (in units $e=1, \hbar=1$ ) and $\hbar=137$ (in units $e=1, c=1$ ), correspondingly. These deformations have an analytic sense of p-adic convergent series. The number 137 has a very interesting geometric sense,

$$
137=11^{2}+4^{2}=| \pm 4 \pm 11 i|^{2}=| \pm 11 \pm 4 i|^{2}
$$

so, $\sqrt{137}$ is the hypotenuse length of a triangle with other sides of lengths 11 and 4 . These relations indicate on a secondary, composed, emergent nature of quantum theory and spatial relativity. Octagon with sites at the complex numbers has horizontal and vertical sites of length 8 and other sites with length

$$
\sqrt{2(11-4)^{2}}=\sqrt{98}=\sqrt{100-2}=10\left(1-\frac{1}{100}+\ldots\right)=9.8995
$$

A sector of a circle with a length of 10 and a chord $\sqrt{98}$ for the angle $\alpha$ and radius $r$ satisfies the following equations:

$$
\begin{aligned}
& \alpha r=10, r \sin \frac{\alpha}{2}=\frac{7}{\sqrt{2}} \Downarrow \\
& \alpha=\frac{10}{r}, r \sin \frac{5}{r}=\frac{7}{\sqrt{2}}, r=20.33, \alpha=0.4919
\end{aligned}
$$

The Babylonians used a base 60 number system which is still used for measuring time 60 seconds in a minute, 60 minutes in an hour - and for measuring angle - 360 degrees in a full turn. The base 60 number system has its origin in the ration of the Sumerian mina $(\mathrm{m})$ and Akkadian shekel (s), $m / s \simeq 60=3 \cdot 4 \cdot 5$.

We also can consider base 137 system for fundamental theories. To describe the nuclear physics strong coupling phenomena, we may take $p=13$ as a base. To describe the hadronic physics, valence scale QCD, and graphen strong coupling phenomena, we may take $p=2$ as a base. To describe the weak coupling physics SM $m_{Z}$ scale and MSSM unification scale phenomena, we may take $p=29$ as a base.

There are different opinions about the number of fundamental constants [4]. According to Okun, [5] there are three fundamental dimensionful constants in Nature: Planck's constant, $\hbar$; the velocity of light, $c$; and Newton's constant, $G$. According to Veneziano, there are only two: the string length $L_{s}$ and $c$. According to Duff, there are no fundamental constants at all. Usually $L_{s}=l_{p}$, so, the fundamental area is $L_{s}^{2}=137 l_{s}^{2}$. The value $s_{s}=l_{s}^{2}-$ Stoney area, is more like a fundamental area.

In mathematics we have two kinds of structures, discrete and continuous one. If a physical quantity has discrete values, it might have no dimension. If the values are continuous - the quantity might have a dimension, a unit of measure. These structures may depend on scale, e.g. on macroscopic scale condensed state of matter (and time) is well described as continuous medium, so we use dimensional units of length (and time). On the scale of atoms, the matter has a discrete structure, so we may count lattice sites and may not use a unit of length. If at small (e.g. at Plank) scale space (and/or time) is discrete, then we do not need a unit of length (time) for measuring, there is a fundamental length and we can just count.

Quarks and gluons can break free from their confinement inside protons and neutrons at a temperature of around $200 \mathrm{MeV}$ - the temperature of the Universe a fraction of a second after the Big Bang. We arrived at this figure by combining the results of supercomputer calculations and heavy-ion collision experiments. It puts our knowledge of quark matter on a 
firmer footing. According to the Big Bang model, the very early Universe was filled with quark-gluon plasma, in which quarks and gluons (the carriers of the strong nuclear force) existed as individual entities. The strong force between quarks increases rapidly with distance, that means that the quarks need large amounts of energy to remain free - and therefore the plasma can only exist at extremely high temperatures. When the cosmos was only about a millionth of a second old, it had cooled to the point where quarks and gluons combined to form composite particles such as protons and neutrons. QCD at nonzero temperature and baryon chemical potential plays a fundamental role in description of a number of various physical systems. Two important ones are neutron stars, which probe the low temperature and intermediate baryon chemical potential domain, and heavy ion collision experiments, which explore the region of the high temperature and low baryon chemical potential. There exist low-dimensional theories, such as (1+1)-dimensional chiral Gross-Neveu (GN) type models, which possess a lot of common features with QCD (renormalizability, asymptotic freedom, dimensional transmutation, the spontaneous breaking of chiral symmetry) and can be used as a laboratory for the qualitative simulation of specific properties of QCD at arbitrary energies. Besides temperature and baryon density, there are additional parameters, for instance, an isotopic chemical potential $\mu_{I}$. It allows one to consider systems with isospin imbalance (different numbers of $u$ and d quarks). It is realized, e.g., in neutron stars, heavy-ion experiments, etc.

The thermodynamics of QCD is most conveniently described by the grand canonical partition function [6]

$$
\begin{aligned}
& Z(\alpha, \beta)=\operatorname{tr} e^{-\alpha Q-\beta H}=\int d A d \bar{q} d q \exp \left(-S_{A}-S_{q}\right), \\
& S_{A}=\int_{0}^{\beta} d x_{0} \int_{V} d^{3} x \operatorname{tr}\left(F_{\mu \nu}^{2} / 2\right), \\
& S_{q}=\int_{0}^{\beta} d x_{0} \int_{V} d^{3} x \prod_{f=1}^{N_{f}} \bar{q}_{f}\left(\gamma_{\mu} D_{\mu}-m_{f}-\alpha / \beta \gamma_{0}\right) q_{f}
\end{aligned}
$$

Another form of functional representation is

$$
\begin{aligned}
& Z(\alpha, \beta)=\operatorname{tr}^{-\alpha Q-\beta H}=\int d A d \bar{q} d q \exp \left(-S_{A}-S_{q}\right), \\
& S_{A}=\beta / \alpha \int_{0}^{\alpha} d x_{0} \int_{V} d^{3} x \operatorname{tr}\left(F_{\mu \nu}^{2} / 2\right), \\
& S_{q}=\int_{0}^{\alpha} d x_{0} \int_{V} d^{3} x \prod_{f=1}^{N_{f}} \bar{q}_{f}\left(\left(\gamma_{\mu} D_{\mu}-m_{f}\right) \beta / \alpha-\gamma_{0}\right) q_{f}
\end{aligned}
$$

We may interpret the charge density as a second Hamiltonian: $Q=H_{2}, H_{1}=H$ and consider corresponding classical Nambu's dynamics

$$
\dot{A}=\left\{A, H_{1}, H_{2}\right\}
$$

As a quantum Nambu's dynamics we consider above functional representations.

The partition function depends on the external macroscopic parameters $V, T, \mu$, as well as on the microscopic parameters like masses and the coupling constant. Once the partition function is known, thermodynamic properties such as free energy, pressure, average particle numbers or the thermal expectation value of an operator $A$ readily follow,

$$
F=-T \ln Z, P=-\frac{\partial F}{\partial V},<Q>=-\frac{\partial \ln Z}{\partial \mu}
$$


Note that the functional form is a trace from the evolution operator in imaginary time. For real time the definition is formal. Similarly, it is defined for imaginary chemical potential $i \mu$ and for real $\mu$ the definition is formal. So it is natural to consider Wick rotation for both parameters.

The rich structure of separated energy scales makes quarkonium an ideal probe of confinement and deconfinement. The different quarkonium radii provide different measures of the transition from a Coulombic to a confined bound state. Different quarkonia will dissociate in a medium at different temperatures, providing a thermometer for the plasma. Let us construct a model of a physical system with maximal (Hagedorn) temperature $T_{H}=\beta_{H}^{-1}, T<T_{H}$. The system consists of $N$ identical (noninteracting) subsystems $s_{n}$ each of which can be in $p>1$ states with same energy $\varepsilon$. So the number of states of the system with given energy $E=N \varepsilon$ is $M=p^{N}$ and corresponding entropy is $S=N \ln p$. To different energies $E$ correspond different $N=E / \varepsilon$. The statistical sum of the system is

$$
Z(\beta)=\sum_{E} \rho(E) e^{-\beta E}=\sum_{N} e^{-\left(\beta-\beta_{H}\right) \varepsilon N}, \beta>\beta_{H}=\frac{\ln p}{\varepsilon} .
$$

Note that, for QCD $T_{H} \simeq 150 \mathrm{MeV}$. Quantum computers (quanputers) will become commercially interesting for systems with $N>100$.

Let us consider potential $V$ and corresponding force $F$ of an elementary charge $e$ in a scalar field $\varphi$ generated in $D$-dimensional euclidean space by corresponding point chargesource $g$ defined as a solution of the following equation:

$$
\begin{aligned}
& \Delta_{D} \varphi=g \delta^{D}(x), \\
& \int d x^{D} \Delta_{D} \varphi=\int d S_{D-1} \nabla \varphi=\Omega_{D} r^{D-1} \varphi^{\prime}(r)=g \Downarrow \\
& \varphi(r)=-\frac{1}{(D-2) \Omega_{D}} \frac{g}{r^{D-2}}, D \neq 2 \\
& D=2: 2 \pi r \varphi^{\prime}(r)=g \Rightarrow \varphi(r)=\frac{g}{2 \pi} \ln \frac{r}{r_{0}}, \\
& V_{D}=-\frac{1}{(D-2) \Omega_{D}} \frac{e g}{r^{D-2}}, F_{D}=-\nabla V_{D}=-\frac{e g}{\Omega_{D} r^{D-1}}, \Omega_{3}=4 \pi \\
& V_{2}=\frac{e g}{2 \pi} \ln \frac{r}{r_{0}}, F_{2}=-\frac{e g}{2 \pi r}
\end{aligned}
$$

For Newton potentials charges $e, g$ play pole of masses, they are, by definition and in correspondence with observations, positive and for $D>2$ we have attraction of masses. For $D \leq 2$ we have attraction and confinement. From Newton potential we obtain the Coulomb one if we take imaginary masses $m=i e_{ \pm}$.

Let me draw the following scenario of confinement and particle production in QCD. In classical gluodynamics (in the simplest case, $A_{0}=0$ and finite energy assumption) particlelike solutions, monopoles, can not be due to scale (conformal) invariance. For nontrivial asymptotic $A_{0}$, we may have monopole states. In quantum gluodynamics we have not conformal invariance beyond the renormdynamic fixed points and monopoles can exist. In (one charge) quantum gluodynamics we have the trivial ultraviolet fixed point at $g=0$ and nontrivial infrared fixed point at some $g=g_{c}$. The Higher energy multiparticle production processes follow the scenario: higher energy quarks and gluons (perturbatively) produce lower energy gluons and quarks until the intermediate energy-scale where running coupling constant reaches the selfdual (fixed) point beyond which monopoles start to produce. Later at the valence quark energies-scales, (at which $\alpha_{s}=2$,) monopoles become unstable and decay into hadrons. 
Acoustic cavitation-the formation and implosive collapse of bubbles-occurs when a liquid is exposed to intense sound. Cavitation can produce the emission of light, or sonoluminescence. The concentration of energy during the collapse is enormous: the energy of an emitted photon can exceed the energy density of the sound field by about twelve orders of magnitude, and it has long been predicted that the interior bubble temperature reaches thousands of degrees Kelvin during collapse. Sonoluminescence refers to that remarkable phenomenon in which a small bubble of air injected into a container of water and suspended in a node of a strong acoustic standing wave emits light. More precisely, if it is driven with a standing wave of about $20,000 \mathrm{~Hz}$ at overpressure of about $1 \mathrm{~atm}$, the bubble expands and contracts in concert with the wave, from a maximum radius $R \sim 10^{-3} \mathrm{~cm}$ to a minimum radius of $r \sim 10^{-4} \mathrm{~cm}$. Note that $R / r \sim 10$ one order of magnitude as in QCD with size for hadrons $R \sim 10^{-13} \mathrm{~cm}$ and perturbative size $r \sim 10^{-14} \mathrm{~cm}$. Exactly at a minimum radius roughly 1 million optical photons are emitted, for total energy liberated of $10 \mathrm{MeV}$.

Let as consider the following formula:

$$
\frac{1}{1-x}=(1+x)\left(1+x^{2}\right)\left(1+x^{4}\right) \ldots,|x|<1 .
$$

which can be proved as

$$
\begin{aligned}
& p_{k} \equiv(1+x)\left(1+x^{2}\right)\left(1+x^{4}\right) \ldots\left(1+x^{2^{k}}\right)=\frac{1-x^{2^{(k+1)}}}{1-x}, \\
& \lim _{k \rightarrow \infty} p_{k}=1 /(1-x) .
\end{aligned}
$$

The formula (12) reminds us the boson and fermion statsums:

$$
Z_{b}=\frac{\sqrt{x}}{1-x}, Z_{f}=\frac{1+x}{\sqrt{x}}, x=\exp (-\beta \omega)
$$

and can be transformed in the following relation:

$$
Z_{b}(\omega)=Z_{f}(\omega) Z_{f}(2 \omega) Z_{f}(4 \omega) \ldots
$$

Indeed, [7]

$$
\begin{aligned}
& Z_{b}(\omega)=\frac{\sqrt{x}}{1-x}=x^{a} Z_{f}(\omega) Z_{f}(2 \omega) Z_{f}(4 \omega) \ldots, \\
& a=1+\left(1+2+2^{2}+\ldots\right)=1+\frac{1}{1-2}=0,|2|_{2}=1 / 2<1 .
\end{aligned}
$$

By the way, we have an extra bonus! We see that the fermi content of the boson wears the p-adic sense. The prime $p=2$, in this case. Also, the vacuum energy of the oscillators has the p-adic sense.

What about other primes $p$ ? For the finite fields,

$$
\begin{aligned}
& z_{n}(p)=\exp (2 \pi i n / p), n=0,1, \ldots, p-1, \sum_{n} z_{n}=0 \\
& Z_{p}(\beta)=\sum_{n=1}^{p-1} \exp \left(-\beta E_{n} / \hbar\right), E_{n}=2 \pi \hbar(n+a) \\
& Z_{p}(-i / p)=0, p=2,3,5, \ldots 13 \ldots 29 \ldots 137 \ldots
\end{aligned}
$$

The Riemann zeta function $\zeta(s)$ is defined for complex $s=\sigma+i$. All complex zeros, $s=\alpha+i \beta$, lie in the critical stripe $0<\sigma<1$, symmetrically with respect to the real axis and critical line $\sigma=1 / 2$. So it is enough to investigate zeros with $\alpha \leq 1 / 2$ and $\beta>0$. These zeros are of three types, with small, intermediate and big ordinates. The Riemann 
hypothesis states that the (non-trivial) complex zeros of $\zeta(s)$ lie on the critical line $\sigma=1 / 2$. The Riemann hypothesis (RH) is a central problem in Pure Mathematics due to its connection with Number theory and other branches of Mathematics and Physics.

Let us consider the following finite approximation of the Riemann zeta function:

$$
\begin{aligned}
& \zeta_{N}(s)=\sum_{n=1}^{N} n^{-s}=\frac{1}{\Gamma(s)} \int_{0}^{\infty} \frac{\left(e^{-t}-e^{-(N+1) t}\right) t^{s-1} d t}{1-e^{-t}}=\zeta(s)-\Delta_{N}(s), \text { Re } s>1 \\
& \zeta(s)=\frac{1}{\Gamma(s)} \int_{0}^{\infty} \frac{t^{s-1} d t}{e^{t}-1}, \Delta_{N}(s)=\frac{1}{\Gamma(s)} \int_{0}^{\infty} \frac{t^{s-1} e^{-N t} d t}{e^{t}-1}
\end{aligned}
$$

Another formula, which can be used on critical line, is:

$$
\zeta(s)=\left(1-2^{1-s}\right)^{-1} \sum_{n \geq 1}(-1)^{n+1} n^{-s}=\frac{1}{1-2^{1-s}} \frac{1}{\Gamma(s)} \int_{0}^{\infty} \frac{t^{s-1} d t}{e^{t}+1}, \text { Re } s>0
$$

Corresponding finite approximation of the Riemann zeta function is:

$$
\begin{aligned}
& \zeta_{N}(s)=\left(1-2^{1-s}\right)^{-1} \sum_{n=1}^{N}(-1)^{n-1} n^{-s} \\
& =\frac{1}{1-2^{1-s}} \frac{1}{\Gamma(s)} \int_{0}^{\infty} \frac{t^{s-1}\left(1-\left(-e^{-t}\right)^{N}\right) d t}{e^{t}+1}=\zeta(s)-\Delta_{N}(s), \\
& \Delta_{N}(s)=\frac{1}{\Gamma(s)} \int_{0}^{\infty} d t \frac{\left.t^{s-1}\left(-e^{-t}\right)^{N}\right)}{e^{t}+1} \sim \pm N^{-s}
\end{aligned}
$$

at a (nontrivial) zero of the zeta function, $s_{0}, \zeta_{N}\left(s_{0}\right)=-\Delta_{N}\left(s_{0}\right)$. In the integral form, dependence on $N$ is analytic and we can consider any complex valued $N$.

It is interesting to see dependence (evolution) of zeros with $N$. For the simplest nontrivial integer $N=2, \zeta_{2}(s)=\left(1-2^{1-s}\right)^{-1}\left(1-2^{-s}\right)$, we have zeros at $s_{n}=2 \pi i n / \ln 2, n=0, \pm 1, \pm 2, \ldots$, $2 \pi / \ln 2=9.06$. In the interval $I m s_{n} \in(0,100)$ we have 10 nontrivial zeros. The first nontrivial zero of the zeta function, by Mathematica, is: $s_{1}=1 / 2+14.1347 i$. The last zero in the interval $I m s_{n} \in(0,100)$ is: $s_{29}=1 / 2+98.8312 i$.

\section{References}

[1] N. Koblitz, p-adic numbers, p-adic analysis, and Zeta-functions (Springer-Verlag, New York, 1977)

[2] G. Stoney, Phil. Mag. 11, 381-391 (1881)

[3] M. Planck, Ann. d. Phys. 1, 69 (1900)

[4] M. Duff, L. Okun, G. J. Veneziano, High Energy Phys. 0203, 023 (2002)

[5] L. Okun, Sov. Phys. Usp. 34, 818 (1991)

[6] M. Le Bellac, Thermal Field Theory (Cambridge University Press, Cambridge, 1996)

[7] N. Makhaldiani, Physics of Particles and Nuclei 49, 961 (2018) 